\title{
Paradiesgarten, himmlische Stadt
}

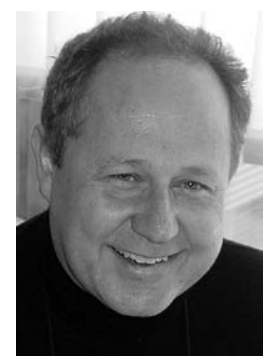

Prof. Dr. med. Manfred Wildner
Korrespondenzadresse

Prof. Dr. med. Manfred Wildner

Bayerisches Landesamt für Lebensmittelsicherheit

Veterinärstraße 2, 85764 Oberschleißheim

Manfred.Wildner@Igl.bayern.de

Bibliografie

DOI http://dx.doi.org/10.1055/s-0042-124516

Gesundheitswesen 2017; 79: 5-6 | @ Georg Thieme Verlag KG

Stuttgart · New York I ISSN 0941-3790
Die Gärten am Rande der persischen Steppe müssen geradezu verzaubert haben: „Paradies“ wurden sie genannt, umgeben von in der Regel hohen Mauern boten sie Raum für Musik und Spiel, Essen und Trinken und wohl auch darüber hinaus die drittwichtigste Sache der Welt. Das griechische Wort paradeisos (Bedeutung: Tiergarten, Park) stammt ebenso wie das hebräische „pardes“ von der altiranischen Bezeichnung „pairi-daeza“ ab, welches für einen umgrenzten (Garten-)Bereich verwendet wurde. In der jüdisch-christlichen Paradies-Tradition wurde der Apfel vom Baum der Erkenntnis dann auch mit Sexualität gleichgesetzt, deren Genuss paradoxerweise zum Verlust des Paradieses führte. Dies ist der hebräischen Nebenbedeutung von Erkennen, welche auch ein Ausdruck für die oben angeführte Drittwichtigste Sache der Welt ist, zuzuschreiben. Wir dürfen es getrost bei der wortgetreuen Wiedergabe des Apfelbaumes als „Baum der Erkenntnis von Gut und Böse“ belassen. Als Ursache des verlorenen Paradieses wäre, plausibler und dem Text näher ein menschheitsgeschichtlich erster Schritt in Richtung intellektuell-moralischer Unterscheidung und Bewertung.

Den Verlust eines unschuldigen, gleichsam paradiesischen Nichtwissens beklagen bisweilen auch romantische Naturutopien. Hier liegt der Verdacht nahe, dass uns in die Vergangenheit projizierte menschliche Zukunftshoffnungen gegenübertreten. Wir tun gut daran, uns der Wurzel des Paradiesgartens zu erinnern: nämlich eines durch menschlichen Willen, menschliches Wissen und menschliche Anstrengung geschaffenen Kulturraumes in Abgrenzung zur oftmals große Härten mit sich bringenden „natürlichen“ Umwelt. Doch trägt diese Abgrenzung von Natur und Kultur überhaupt? Ist nicht des Menschen Kultur seine Natur (Konrad Lorenz)? Sind wir Menschen nicht auf Kultur angewiesen, um nicht als „Mängelwesen“ zu erscheinen (Arnold Gehlen)? Hier ließe sich auch noch Vieles aus der ökonomischen und soziologischen Literatur ergänzen. Vielleicht kann man in Bezug auf menschliche Kulturräume in diesem Sinne sogar von einer Natur 2.0 oder „Next Nature“ sprechen (www.nextnature. net)? So plädiert z. B. das Next-Nature-Netzwerk für eine be- wusste Vernetzung von (natürlicher) Natur und vom Menschen geschaffener Kultur, welche eine Balance zwischen Biologie und Technologie im Dienst am Menschen hält.

Für die menschliche Gesundheit scheint diese hoffnungsfrohe Deutung in mancher Hinsicht zuzutreffen: Gesundheit ist nicht so sehr ein natürliche Zustand als vielmehr auch und ganz wesentlich das Ergebnis menschlicher Anstrengung (Heinrich Schipperges). Die nur 50:50 Chance für neugeborene Kinder noch am Beginn des 20. Jahrhunderts, das Erwachsenenalter zu erreichen, spricht eine deutliche Sprache. Wie diese Anstrengungen aussehen? Sie reichen von den oft religiös inspirierten Barmherzigkeitsidealen, der politischen Einschränkung von Gewalt und Ausbeutung über die Verbesserung der körperlichen Ernährung und Hygiene bis zur Entfaltung der geistigen Potentiale der Menschheit. Diese Entfaltung reicht von der, von vergleichsweise liberalen Ideen unterstützten Gründung von Universitäten - erstmals wird für das Jahr 737 in Tunis eine islamische Universitätsgründung beschrieben - bis zu den Bemühungen um Aufklärung und Vernunft, um Freiheit, Gleichheit und Brüderlichkeit und in der Moderne zu den oft zitierten, Gesundheit und das Gesundheitswesen betreffenden, Erklärungen von Ottawa (1986), Tallin (2008) und zuletzt Wien (2016).

Doch ist wirklich der Garten, oder modern übersetzt die Idylle des ländlichen Raums, der Ziel-Ort menschlicher Sehnsucht und die Quelle gelingenden Daseins? Begreift man den Paradiesgarten jüdisch-christlicher Prägung (Genesis Kap. 2 und 3) als menschlich geschaffenen Kulturraum, so überrascht nicht, dass er auf dem weiteren Menschheitsweg mit der Vision einer himmlischen Stadt (Offenbarung 21) ergänzt wurde. Städte als kulturelle Verdichtungsräume, als Orte der Begegnung und des Austauschs, der wirtschaftlichen und menschlichen Entwicklungschancen - „Stadtluft macht frei“, diese Assoziation ist bis heute gültig. Dass gleichzeitig auch diese Vorstellung von Stadt utopische Züge hat, zeigen die im 20./21. Jahrhundert weltweit entstehenden Metropolen und Mega-Cities: zivilisatorische Mischformen von baulichen Wüs- 
ten („Grey“ Cities) und hoffnungsvollen städtischen Oasen mit Grünund Wasserflächen („Green“ and „Blue“ Cities).

Dass Utopien und Dystopien eng beisammen liegen können, gilt für erhoffte himmlische Städte offenbar ebenso wie für (verlorene) Paradiesgärten. Vor dem Hintergrund dieser Doppelgesichtigkeit menschlicher Kulturräume, die im besonderen Maß für die bebaute städtische Umwelt gilt, hat die Weltgesundheitsorganisation (WHO) die Thematik von Gesundheit und Urbanisierung zu einer prioritären Herausforderungen erklärt. Eine konkrete Annahme dieser Herausforderung findet sich im Gesunde-Städte-Netzwerk der WHO, welches im 5-jährigen Rhythmus prioritäre Themen benennt und strategische Hilfestellungen für gesundheitsorientierte Entwicklungen gibt. Dieser Ansatz wurde unter der Begrifflichkeit Urban Health/ Stadtgesundheit neu belebt [1,2]. Wie dies für die territoriale Stadtentwicklung im Konkreten aufgegriffen werden kann, ist u. a. Gegenstand der Leipzig Charta zur nachhaltigen europäischen Stadt (2007). Die positiven Auswirkungen von Grünflächen auf die Gesundheit sind empirisch belegt [3]. Besondere Bedeutung wird in diesem Zusammenhang einer integrierten Stadtplanung beigemessen. Ein Beispiel dafür gibt „One New York City: The Plan for a Strong and Just City" [4,5]. Als Kernthema kristallisiert sich die Verkehrsplanung heraus: Ziel ist eine kompakte städtische Bauweise, welche einerseits Flächen einspart, andererseits Möglichkeiten für öffentlichen Raum schafft und zum Dritten eine körperlich aktivierende Verkehrsinfrastruktur ermöglicht (Öffentlicher Nahverkehr, Fahrradwege usw.). Hinzu kommen Aspekte besserer sozialer Vernetzung, von mehr Chancengerechtigkeit und in der Summe von mehr Lebensqualität. Die Verantwortungsübernahme für solche verbesserten Lebensumwelten wird "Jenseits von Eden" von uns Menschen selbst als gute „Stewards“ einzufordern sein.

Healthy Cities, Green Cities, Blue Cities, Smart Cities, Neighbourhood Walkability oder auch Feinstaubgrenzwerte - die zivilisatorischen Anstrengungen sind unübersehbar und zu begrüßen. Inwieweit hier auch Balancen zwischen dem ländlichen und dem städtischen Umfeld, gleichsam eine Balance des alten Paradiesgartens mit der himmlischen Stadt, berücksichtigt werden müssen, ist noch zu erforschen. Bieten nicht Städte auch Chancen für mehr medizinische Versorgungsqualität durch erhöhte Spezialisierung, für gesundheitsbezogene Innovation in Strukturen und Abläufen, für (universitäre) Forschung und Lehre? Und kann möglicherweise die Abgrenzung zwischen ländlichen und städtischen Räumen durch die virtuelle Vernetzung und Distanzverkürzung über telemedizinischen Anwendungen teilweise aufgehoben werden? Die Herausforderung des Erhalts der ärztlichen Versorgung im ländlichen Raum ist eines der dabei zu bearbeitenden Themen. Doch gibt es nicht auch ganz andere Bedrohungen durch eine zunehmend urbanisierte Menschheit, sei es in Form von regionaler Umweltverschmutzung, sei es in Form von globalen Belastungen wie Klimawandel, Ernteausfällen und Armutsmigration? Ist nicht auch der ökologische Kollaps von ganzen Zivilisationen eine realistische Annahme [6]? Der „One Health“-Ansatz erkennt zunehmend die Interdependenz von menschlicher Gesundheit mit Aspekten der Ökologie und der Gesundheit der Tiere an (Manhattan Principles, 2004), bisweilen wird auch schon von einer planetaren Gesundheit gesprochen [7]. Bezogen auf Gesundheit und das medizinische Versorgungssystem wird vor diesem Hintergrund der Ruf nach einer guten „Stewardship“ laut: Hierunter versteht die WHO ein kluges Regierungshandeln, welches im Wettstreit stehende In- teressen in Hinblick auf bestmögliche und nachhaltige Gesundheitschancen ausbalanciert. Dabei werden Werte wie Chancengerechtigkeit, Zugang, Inklusion, Qualität und Patientenrechte berücksichtigt (http://www.who.int/healthsystems/stewardship/en/).

Mit den vielfältigen gesellschaftlichen und humanitären Fragen menschlicher Gesundheit auch als menschlicher Leistung beschäftigen sich auch die Beiträge in dieser Ausgabe: mit den Perspektiven der Universitätsmedizin, dem Konsum psychotroper Substanzen während der Ausbildung, den regionalen Unterschieden der Versichertenpopulation, den Assoziationen von Krankenversicherung und soziodemografischen Merkmalen, der Optimierung der kommunalen Gesundheitsversorgung, dem Gate-Keeping-Ansatz im Gesundheitswesen in China, der Teilhabe als Ziel von Sozialmedizin und Pflege, der Stufendiagnostik nach der Richtlinie des GBA und mit Kostenansätzen zur monetären Bewertung von Leistungen.

Dass vor Utopien auch gewarnt werden muss, hat uns unter anderem Karl Popper ans Herz gelegt: „Der Versuch, den Himmel auf Erden einzurichten, erzeugt stets die Hölle“ [8]. Die traurige historische Beweisführung wurde von den verschiedenen Zerrbildern staatlicher Ordnung im 20. Jahrhundert eindrücklich geliefert, als Folgen der Heils-Doktrinen Hitlers, Stalins, Pol Pots und weiterer totalitärer Herrscher. Ob Paradiesgarten oder himmlische Stadt oder gar etwas Drittes - vielleicht unser Planet im Ganzen -, der Schlüssel zum Paradies dürfte in der Annahme der Welt als das, was sie ist, liegen. Mit ihrer Schönheit und eben auch ihren schmerzlichen Herausforderungen. Daseinsbewältigung und Daseinsverbesserung verlangen zunächst die Annahme der realen Herausforderungen, ein oft mühsam geschaffenes Wissen und eine begleitende, oft nicht weniger mühsam erarbeitete individuelle und kollektive Weisheit. So ist der größte Reichtum unserer Welt aus dieser Perspektive vor allem unsere menschliche Energie, in städtischen wie in ländlichen Regionen - und Gesundheit ist einer der wesentlichen Schlüssel zu dieser Energie [9].

\section{Literatur}

[1] Fehr R, Fertmann R, Stender KP et al. Stadtgesundheit (Urban Health) - Eine Blickfelderweiterung am Beispiel Hamburgs. Gesundheitswesen 2016; 78: 498-504

[2] Möbus S, v. Mittelstädt G. Metropolis - Gesundheit anders denken. Gesundheitswesen 2016; 78: 495-497

[3] Gascon M, Triguero-Mas M, Martinez D et al. Residential green spaces and mortality: A systematic review. Environ Int 2016; 86: 60-67

[4] Sallis JF, Bull F, Burdett R et al. Use of science to guide city planning policy and practice: How to achieve healthy and sustainable future cities. Lancet 2016; 388: 2936-2947

[5] De Blasio B. Healthier neighbourhoods through healthier parks. Lancet 2016; 388: 2850-2851

[6] Diamond J. Collapse: How societies choose to fail or succeed. New York: Viking; 2004

[7] Horton R, Beaglehole R, Bonita R et al. From public to planetary health: A manifesto. Lancet 2014; 383: 847

[8] Popper K. Die offene Gesellschaft und ihre Feinde. Band II. Tübingen: Verlag Mohr/Siebeck; 1992: S. 277

[9] Mahler $\mathrm{H}$. The Meaning of ,, health by all of the year 2000“. World Health Forum 1981; 2 : 5-22 